Journal of Computer Science 8 (10): 1718-1729, 2012

ISSN 1549-3636

(C) 2012 Science Publications

\title{
Optical Code-Division Multiple-Access and Wavelength Division Multiplexing: Hybrid Scheme Review
}

\author{
Isaac A.M. Ashour, Sahbudin Shaari, \\ P. Susthitha Menon and Hesham A. Bakarman \\ Institute of Microengineering and Nanoelectronics (IMEN), \\ Photonic Technology Laboratory, \\ Universiti Kebangsaan Malaysia, 43600 UKM Bangi, Selangor, Malaysia
}

\begin{abstract}
Problem statement: Hybrid Optical Code-Division Multiple-Access (OCDMA) and Wavelength-Division Multiplexing (WDM) have flourished as successful schemes for expanding the transmission capacity as well as enhancing the security for OCDMA. However, a comprehensive review related to this hybrid system are lacking currently. Approach: The purpose of this paper is to review the literature on OCDMA-WDM overlay systems, including our hybrid approach of onedimensional coding of SAC OCDMA with WDM signals. In addition, we present an additional review of other categorios of hybrid WDM/OCDMA schemes, where codes of OCDMA can be employed on each WDM wavelength. Furthermore, an essential background of OCDMA, recent coding techniques and security issues are also presented. Results: Our results indicate that the feasibility of transmitting both OCDMA and WDM users on the same spectrum band can be achieved using MQC family code with an acceptable performance as well as good data confidentiality. In addition, the WDM interference signals can be suppressed properly for detection of optical broadband CDMA using notch filters. Conclusion: The paper provides a comprehensive overview of hybrid OCDMA-WDM systems and can be used as a baseline study for other scientists in the similar scope of research.
\end{abstract}

Key words: Wavelength-Division Multiplexing (WDM), Optical Code-Division Multiple-Access (OCDMA), optical communications

\section{INTRODUCTION}

Emerging of wireless CDMA telephony in the 1980s with tremendous benefits of CDMA such as a merit of Spread Spectrum (SS) pushes some researchers to transfer principles and advantages of CDMA into optical communication networks (Viterbi, 1979; Pickholtz et al., 1982; Hata et al., 2006; Hui, 1985; Tamura et al., 1985). The first experiment that demonstrates the feasibility of implementing CDMA thought into the optical domain was in 1986 by (Prucnal et al., 1986). Then Salehi employed CDMA techniques in optical systems and analyzed their performances (Salehi, 1989; Salehi and Brackett, 1989). Up till now, interest in applications of OCDMA has been constantly rising during recent decades (Hamam, 2006).

The nature of OCDMA in security has been concerned for some application of enterprise and military networks. To increase the security degree, the hybrid system of both OCDMA and WDM has been studied, in addition to improve the capacity of simultaneous users. This hybrid scheme is classified into: (1) OCDMA channels-over-WDM, whereby WDM is employed as a medium, (2) In-band transmission of both OCDMA and WDM signals. Most previous reviews in this field focus on pure OCDMA systems and optical coding through their applications. In contrast to the earlier work, this paper reports an essential review of OCDMA systems and its related security. In addition, we survey the current trends in hybrid OCDMA and WDM schemes, including the type of OCDMA coding, security enhancement as well as the systems performance.

Optical CDMA Background: Various multiple access techniques have been developed for optical fiber communications. This can provide a large bandwidth by exploitation of the optical medium capacity. Then, many channels are combined together over a single fiber link. There are three main ways to employ the

Corresponding Author: Isaac A.M. Ashour, Photonic Technology Laboratory, Institute of Microengineering and Nanoelectronics (IMEN), Universiti Kebangsaan Malaysia, 43600 UKM Bangi, Selangor, Malaysia 
multiplexing technique in optical networks. TimeDivision Multiple Access (TDMA) scheme assigns users to transmit data over a dedicated time slot from a number of accessible slots. However, TDMA suffers from a hard limitation of the number of users that is based on the availability of this resource. The second technique is Wavelength-Division Multiple Access (WDMA). Systems that operate by WDM assign a unique wavelength from a number of available wavelengths. On the other hand, in OCDMA, each user has a unique code as an assignment address that spreads over a relatively wide bandwidth. This specific code is modulated and then a message signal is transmitted at an arbitrary time to an intended receiver, which can match the correct code to recover the encoded information. The main OCDMA and WDM systems have been of widespread implementation for local and metro access network (Fouli and Maier, 2007; Wang, 2008). This is because OCDMA systems provide users both simultaneous and asynchronous access to networks with high security (Ashour et al., 2011a; Salehi and Brackett, 1989) and WDM systems supply a relatively high transmission capacity (Agrawal, 2002).

Advantages and challenges of OCDMA: There are some features which make OCDMA technology to be an attractive solution for next-generation of broadband communications. The principle of OCDMA multiplexing leads to support of a larger channel count than other techniques, allows asynchronous transmission with efficient access and enhances information security potentially in the network. Furthermore, it has employment of simplified network control and management, multi-class traffic with different formats and bit rates and can be easily upgraded in terms of its architecture. Moreover, simpler and cheaper configurations can be implemented and produced based on employing incoherent sources. OCDMA's robustness in hostile conditions can be provided by using well-designed special codes with fair division of bandwidth (Fouli and Maier, 2007; Wang, 2008; Leeson et al., 2009).

Despite these potential advantages, there are some issues that challenge this technology. For instance, multiple-user interference generates beat noise that degrades the system performance, especially when optical pulses are transmitted with close wavelengths. In addition, for spectrally coded OCDMA, the broadband light sources which are currently available have either an inadequate generated intensity or the device is expensive (Stok and Sargent, 2002).

Fundamentals of OCDMA: The general principle of coding and decoding of OCDMA is clarified. In coding operation, the spectrum of data signal is broadened as spread spectrum designation. Each user has been assigned to some chips of the code sequences to share the same transmission line using power splitters or combiners. This operation can be performed in the optical-domain and/or in the space-domain as well. Decoders at the receiver recognize a target code by employing match filtering.

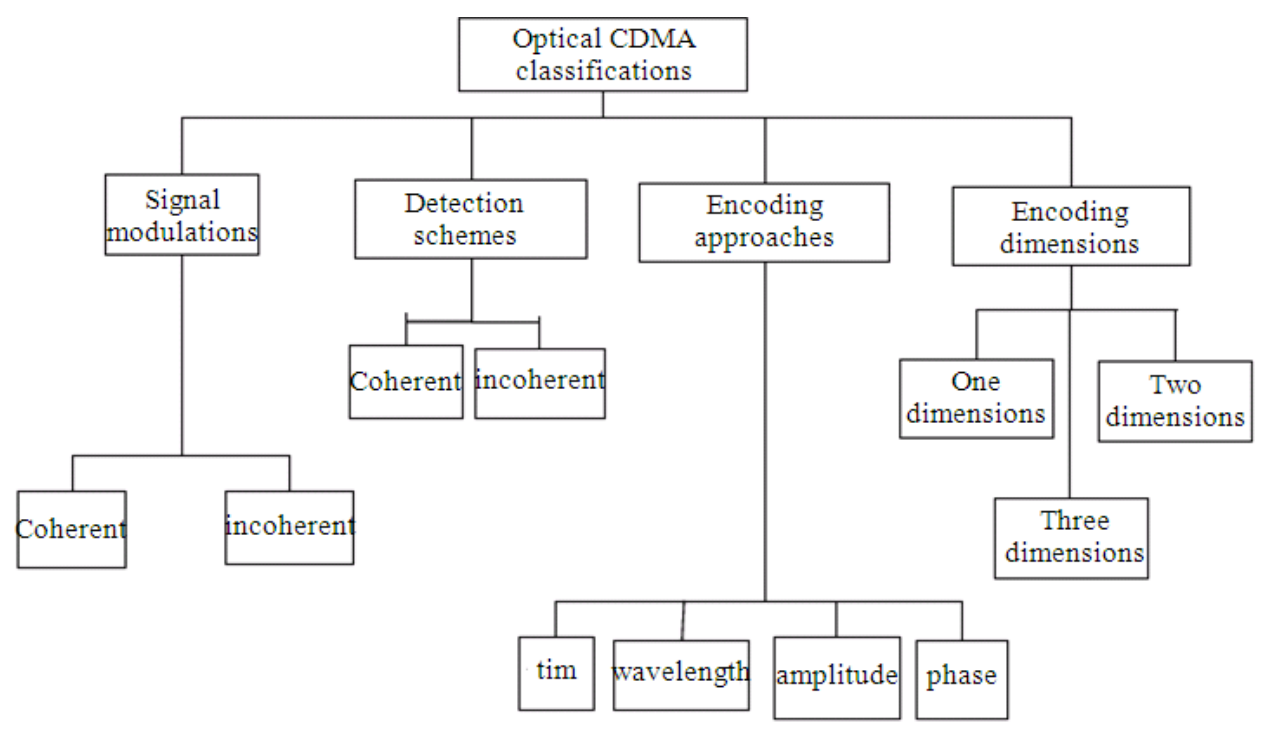

Fig. 1: Optical CDMA system classifications 
It is important to produce high auto-correlations of desired codes, while undesired codes produce crosscorrelation with low-level power. Usually, crosscorrelations are presented between two different codes. An excellent code design has a relatively high code length with high auto-correlation for many users. Finally, electrical thresholding is performed to recover the original data (Fouli and Maier, 2007; Wang, 2008).

Figure 1 shows the summary of OCDMA classifications. Two categories of OCDMA that are classified in terms of signal modulation and detection schemes are coherent and incoherent systems. In a coherent OCDMA scheme, the signal modulation is based on optical phase coding which is often derived from a highly coherent source such as a Mode-Locked Laser (MLL) and detection at a receiver includes knowledge of the phase information of carriers. In other words, the decoded user's data is recovered by applying coherent reconstruction of the signal field. These schemes allow the use of an ultra-short optical pulse for either spectrally coded time spread (SPECTS) by high resolution phase in the encoder/decoder (Etemad et al., 2005) or Spatial Light Phase Modulator (SLPM) (Wang, 2008; Jiang et al., 2005), or directly timeSpread coded by using Superstructure Fiber Bragg Grating (SSFBG) (Wang et al., 2004; 2005; Kitayama et al., 2006) or A Waveguide Grating configuration (AWG) multi-port (Wang et al., 2006). They also allow the usage of bipolar orthogonal coding such as gold sequences (Lee and Kim, 2000). Despite the powerful coherent schemes, these systems are described as complex and very expensive due to the need of a laser source and phase control with accurate adjustment. In contrast, the incoherent systems require more standard techniques of intensity modulation and simpler detection with incoherent source, such as a broadband Amplified Spontaneous Emission (ASE) source, while coherent approaches are based on the optical phase for signal modulation and detection (Lam, 2000). The most common incoherent OCDMA approaches refer to Spectral-Amplitude-Coding (SAC) (Smith et al., 1998; Wei et al., 2001; Aljunid et al., 2004; Ab-Rahman et al., 2009; Sahbudin et al., 2011), temporal (time) spreading (Sotobayashi et al., 2004), spatial coding (Hassan et al., 1995) and Wavelength-Hopping TimeSpreading (WHTS) and two-dimensional code (Yegnanarayanan et al., 2000; Yim et al., 2002; Kwong et al., 2005; Algalbi, 2009). Many of these codes have been developed for either increasing the capacity of systems or enhancing the security of authorized users' data, or both. However, two important factors; cost and performance implications are considered in order to employ incoherent or coherent systems.

If we categorize these systems based on coding optical signals approaches, the six types of coding are available as follows:
- Direct-sequence or temporal coding optical CDMA systems

- $\quad$ Spectral Amplitude Coding (SAC) Optical CDMA systems

- Spectral Phase Coding (SPC) optical CDMA systems

- temporal phase coding optical CDMA systems;

- Two-Dimensional (2-D) spatial or spread space coding optical CDMA systems

- hybrid coding optical CDMA systems (Yin and Richardson, 2007).

In the hybrid coding systems, combined coding techniques that mentioned above is used in one scheme. For example, Wavelength-Hopping Time-Spread (WHTS) encoding is 2-D coding scheme based on merging of spectrum encoding with temporal encoding (Yin and Richardson, 2007). Combintion of the space encoding with previous 2-D coding, Space-Spread WavelengthHopping Time-Spreading encoding (SS-WH-TS) is generated and considered 3-D coding scheme (Yin and Richardson, 2007; Sangin et al., 2000).

If we also categorize the encoding techniques based on the amount of resources of time, wavelength, space and polarization, there are three kinds of optical CDMA systems divided into one-dimensional systems, twodimensional systems and three-dimensional systems as illustrated in Fig. 1 (Yin and Richardson, 2007).

Optical CDMA Security: Security over the optical communications network gives numerous challenges to both network providers and intruders and it experiences the same sensitivities and vulnerabilities as wireless communications (Cederlof and Larsson, 2008). Optical CDMA is a kind of multiplexing for networks of optical communications in which optical pulses are processed by encoding/decoding data applying simple and low cost passive optical components. Its main advantages include asynchronous random access, supporting multiple rates, good compatibility with other multiplexing; TDM and WDM, flexible networking and providing some privacy of transmission information.

OCDMA is a potential system that can boost the security for subscribers. This is because OCDMA encoded signals manifest itself as a noise-like waveform that possibly would not be available to an eavesdropper without knowing and understanding the assigned code of the authorized user (Huang et al., 2010). However, It's important for engineers to know the statement that is nothing of security for a single active user in OCDMA system that employs OOK modulation (Huang et al., 2010; Shake, 2005). Some other advantages belongs to OCDMA systems are 
which contain simplified network control without centralization, increase the versatility of code chips and then increase the degree of spectral efficiency.

The concept of transmission security performance for SAC OCDMA system has been introduced (Bakarman et al., 2008). The correct probability of detected Spectral-Encoding Chip Bandwidth (SECB) pulses in the entire code sequences has been presented by employing several SAC OCDMA codes (Huang et al., 2010). Unipolar OCDMA codes schemes based on Modified Quadratic Congruence (MQC) and Modified Double Weight (MDW) code system enhanced the security with a low cost implementation in comparison to the bipolar ones based on modified Pseudorandom Noise (PN) code (Huang et al., 2010; Bakarman et al., 2009).

Hybrid OCDMA and WDM Overlay System: Hybrid OCDMA and WDM overlay systems emerged in an effort to grow the security for OCDMA systems with may increasing network capacity above the alone system. Inband transmission of both OCDMA and WDM signals under various schemes are reviewed in the following paragraphs. The Summary of hybrid WDM/OCDMA overlay schemes is illustrated in Table 1.

In scheme (1), the first hybrid OCDMA-WDM overlay system has been experimentally demonstrated by Shen and Weiner (2001). The concept of this scheme is that ultra short-pulses are used for spectral encoded/decoded OCDMA. Sub-picoseconed laser pulses that used as short pulses for OCDMA coding make the system more complex technology. The hybrid operation is addressed with high closely spaced WDM users. Focus is at the OCDMA receiver for attenuation of WDM interference for error-free detection. By using a nonlinear fiber thresholder in the decoder, the WDM signals can be filtered and suppressed properly. In (Chang et al., 2005), the same concept was implemented in the optical domain, where OCDMA encoded short pulses spread over time for spectral phase coding. The decoder consists of a Fourier transform for the windowed data signals. The WDMA technique can allow electrical user signals to access the optical networks. Both access approaches in a combined scheme is to achieve high throughputs, resourceful and high-speed access to networks of WAN, greatly faster and further flexible to LANs (Chen, 2005). A matched filter of transmitted pulses includes a conjugate modulator followed by an integrator. Consequently, the signals from both systems can be separated sufficiently based on the perfectly synchronous case for every one of users. Therefore, the effects of thermal noise and adjacent-channel interferences can be neglected. In this hybrid approach, a trade-off between the ratio of bit rates and the total throughput was considered. In a new approach, the time-domain Spectral Phase En/Decoding (SPE/D) OCDMA technique was developed by combining with Group Velocity Dispersion (GVD) (Gao et al., 2010). This new technique was proposed for security enhancement of the secure signal which is hidden into a public channel. A Linearly Chirped Fiber Bragg Grating (LCFBG) and phase modulation for pulse broadening and phase coding, respectively, with 32chip 40-Gchip/s gold codes have been utilized for achieving the stealth channel. Then they are transmitted over an existing WDM system. The proposed scheme for security enhancement was also demonstrated experimentally. It is with much difficulty to intercept the stealth signal from the WDM channel without the spreading of the functional knowledge. The disadvantage of this system was that it depends on the temporal synchronization between encoding and decoding. In order to correctly decode the stealth signal, highly accurate synchronization is required. However, the WDM signal at the stealth channel detection appears like noise in the time domain due to the effect of using LCFBG. Moreover, the public WDM interference was significantly reduced by the optical thresholding. The BER of the stealth channel gradually degrades from $10^{-10}-10^{-2}$ if the received power increases from 13-19 dB.

In scheme (2), the coherent spectral phase encoded OCDMA was applied over an existing WDM network for secure transmission (Wu and Narimanov, 2006). Two signals are used as shown in Fig. 2; a secure signal is encoded and temporally spread to be hidden under a host channel. The purpose of the host channel in this scheme is to provide an ad hoc security enhancement for an encoded signal. Only two channels are considered in the performance analysis of the proposed system; one for the host of WDM network employing OOK and one for a secure $\mathrm{M}$-ary signal at $\mathrm{M}=2$ for the dedicated user in OCDMA. The composite signal is amplified by the EDFA amplifier and hence, the noise from the amplifier due to spontaneous emission is added. Therefore, the secure signal can be completely masked and covered using the amplifier noise as well as the host channel since the average power of this signal is lower than them. Then, this combined signal is fed into the optical fiber link. The nonlinearity effect is assumed to be negligible for a small peak power and the effect of dispersion is also negligible since a compensator is used. The OCDMA en/decoder consists of a coherent spectral phase with direct detection.

The theoretical analysis for both signals gives a clear description for the system performance and also for data confidentiality. 


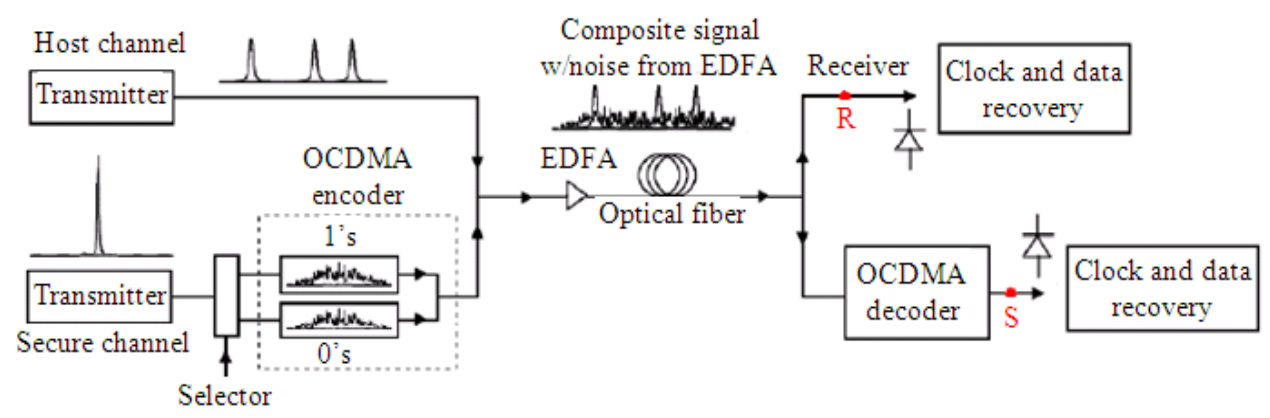

Fig. 2: Schematic diagram of the hybrid system (Wu and Narimanov, 2006)

The Bit-Error-Rate (BER) performance is subject to some factors, such as different code lengths, additive noise in the fiber and operating signal powers, as well as bit rates between secure and host users $(\mathrm{Wu}$ and Narimanov, 2006). The limitation of the performance system exists due to that the amplifier generates effective noise and due to multi-access interference. For security investigation of OCDMA, the covert signal in this security approach could be detected using the following techniques:

- Spectrum analysis: an eavesdropper can look at the power spectrum to confirm secure transmission, but the secure signal's low magnitude and the host channel occupy the same spectrum. This makes an eavesdropper to face difficulties in order to find out the secure transmission

- Monitoring of signal power: using a tunable decoder by an eavesdropper to intercept the stealth signals, in attempt to "brute force" data detection. Since the secure signal occupies the identical frequency spectrum as the public channel, the unwanted public signal is not filtered out as well as the effective noise from the standpoint eavesdropper. In other words, this approach forces an eavesdropper to suffer a lower SNR due to the spreading of public signals while decoding takes place

- Statistical analysis of power fluctuations: the usage of random phase-coding with high number of chips makes the stealth signals to have similar statistics as objected to the amplifier noise present in the network (Wu and Narimanov, 2006). Consequently, the stealth signal is masked by itself naturally by construction and detected by unauthorized users

- Quantitative description: the standard Q factor is to describe the BER/SNR performance of communication networks
In one possible mechanism, using a tunable detector and looking for an increase in the opening of the eye diagram versus the value of the phase of a particular chip (Wu and Narimanov, 2006). But the choice of the "correct" or "wrong" in the case of phase makes large eye opening, consequently, the eavesdropper is subjected to choose the "incorrect" chips at random and spend much time for the correct detection. In conclusion of this approach, the security transmission was enhanced with both cryptographic and steganographic types

In scheme (3), the idea is similar to that of ( $\mathrm{Wu}$ and Narimanov, 2006; Kiurvtso et al., 2007), except the technique of coding. The secure signal is spectrally encoded by implementing optical frequency hopping technique, using tunable delay lines for different frequencies, that is often called (WHTS) (Wu et al., 2006). Then, a dispersive element is utilized for time spreading and then for hiding the OCDMA channel under a public WDM channel. This approach can raise the obscurity for an eavesdropper to detect the data from the secure signal. The stealth signal has a small average power compared with WDM signal. That leads the secure channel to suffer from "crosstalk" interferences. The result indicates that low BER performance of $10^{-9}$ can be achieved with the proper selection of parameters, for example; time spreading, intensity and bit rate for the secure user (Wu et al., 2006).

In scheme (4), a novel coding technique has been proposed for transmission of some channels of both OCDMA and SONET through the same WDM window (Galli et al., 2005). The obtained results from the experiment of the hybrid system show that several OCDMA and a conventional OC-192 OOK channel can be transmitted simultaneously, where OCDMA signals occupy the idle bandwidth of a WDM channel. In the OCDMA approach, a phase-locked frequency such as a Mode-Locked Laser (MLL) source is required with considering the frequency spacing equal to the pulse repetition rate. 


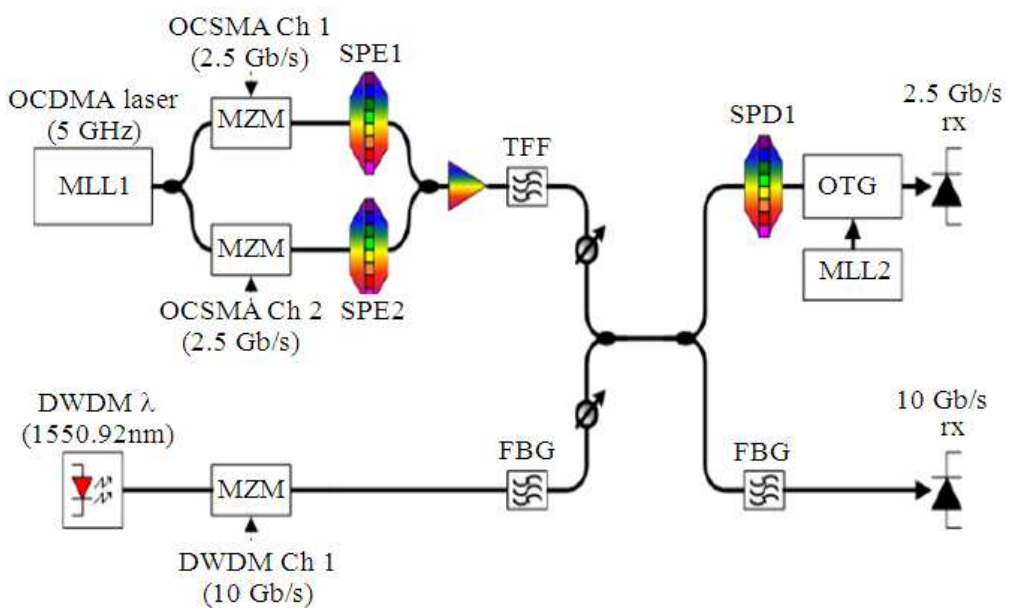

Fig. 3: Both channels of OCDMA and SONET through same WDM window (Galli et al., 2005)

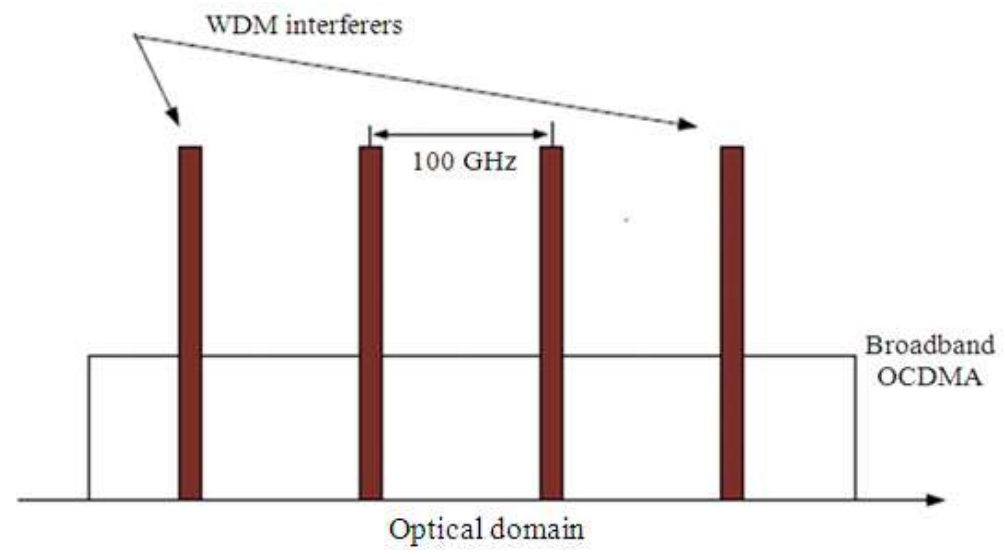

Fig. 4: Hybrid WDM and OCDMA overlay signals in the optical domain

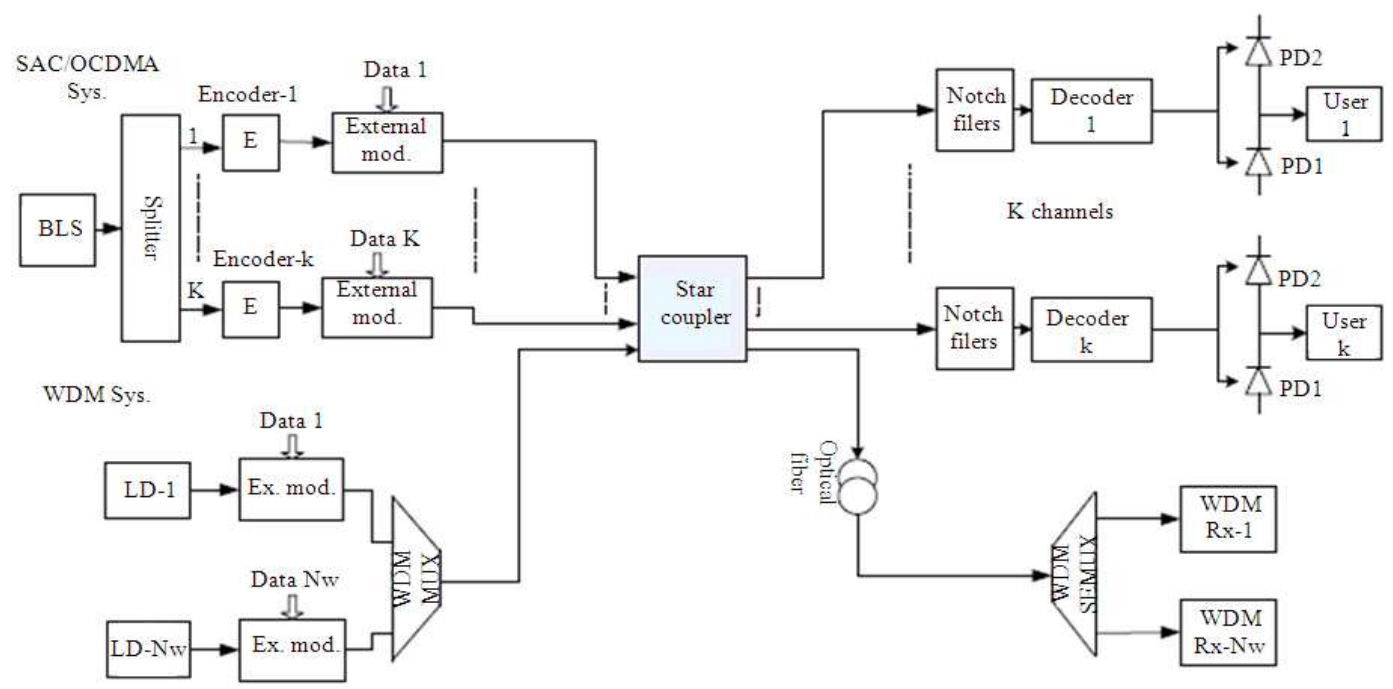

Fig. 5: Block diagram of hybrid SAC OCDMA-WDM overlay system 


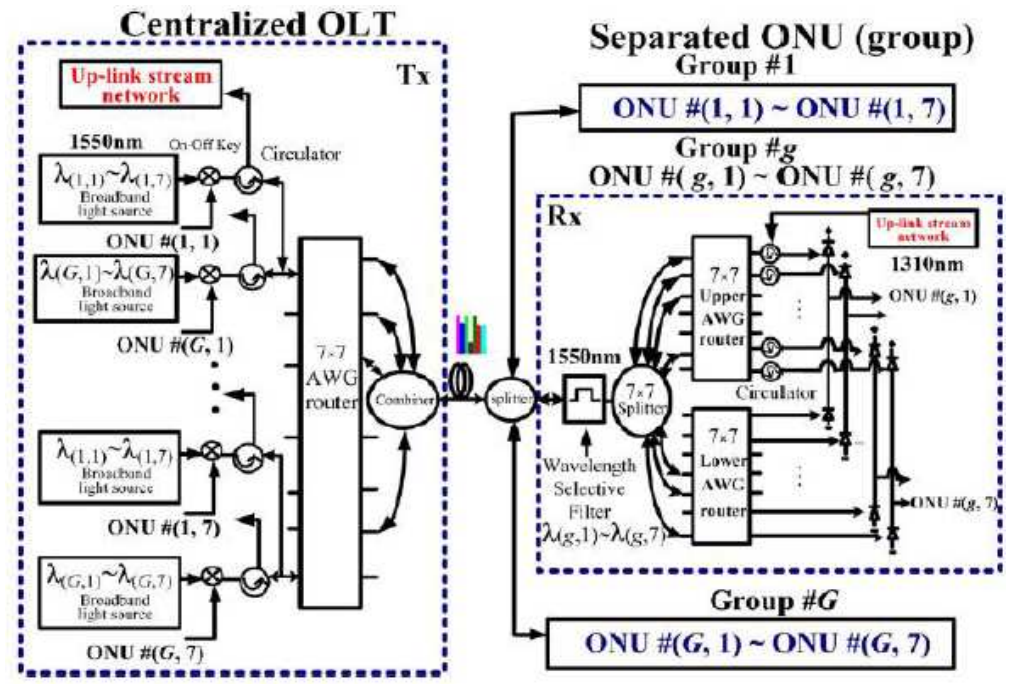

Fig. 6: Hybrid WDM/OCDMA technique over FTTH (Huang et al., 2006)

In addition, optical coders are phase mask modified in order to get simple OOK coding of MLL spectral lines. Particularly, the modification is to allow for: 20 demultiplexed spectral lines, phase encoding of 16 MLL lines using 16 codes length of Hadamard codes and rejecting four central MLL lines using hard filters. It is worth mentioning, the spectral hole in the OCDMA range middle is to use duobinary encoding, hence, modulation the OOK signal at 10 Gbps. Figure 3 illustrates the concept of this hybrid scheme. The experimental results show that the BER is $10^{-9}$ with a 1 $\mathrm{dB}$ power penalty when the OCDMA signal is received at the same (or lower) power than the OC-192 signal (Galli et al., 2005).

It is worth mentioning that techniques of all of the above schemes had not investigated both the performance and security system for conventional codes of SAC OCDMA with WDM signals under a truly hybrid scheme. The SAC OCDMA system would be easily constructed with an additional enhancement for data security by hybridization with WDM system.

In scheme (5), our previous hybrid approach consists of channels of both SAC OCDMA and WDM systems (Ashour et al., 2010; 2011a,b). The MQC family code is the one dimensional code employed for the SAC OCDMA as well as MDW code. WDM signals overlay on code pulses of OCDMA as shown in Fig. 4 and 5. In addition, the WDM interference signals can be suppressed properly for detection of optical broadband CDMA using notch filters. The system performance and security enhancement have been investigated by simulation and theoretical analysis. The results indicate that the feasibility of transmitting both OCDMA and WDM users on the same spectrum band can be achieved with an acceptable performance as well as good data confidentiality (Ashour et al., 2011b). A summary of the hybrid schemes is shown in Table 1.

Hybrid WDM/OCDMA system: in this hybrid scheme, codes of OCDMA can be employed on each WDM wavelength. Recently, this kind of hybrid system has been widely researched because it can support many more users with high data rates. Consequently, several new codes for OCDMA have been proposed and studied over the hybrid architecture.

Yang (2005) has proposed the first WDM and SAC OCDMA system for LANs. The WDM/SAC Balanced Incomplete Block Design (WS-BIBD) code was used to support a larger number of active users by reducing the effect of the PIIN noise (Yang, 2005). Mohammed et al. (2007), the double weight (DW) code family in a hybrid WDM/SAC system has also been studied. However, these systems are not capable of providing flexible and confidential access to optical networks. Therefore, Huang et al. (2006) presented a hybrid WDM/OCDMA system over Fiber To-The-Home (FTTH) networks as shown in Fig. 6. Maximal length sequence (M-sequence) codes were exploited by merit of the cyclic properties of Arrayed Waveguide Grating (AWG) routers (Huang et al. 2006). For more developed systems, the novel OCDMA en/decoders for different WDM channels have been proposed in ( $\mathrm{Pu}$ et al., 2007) where a phase mask was applied on a Super-Structure fiber-Bragg-Grating (S-SBG) based en/decoder to construct A Wavelength-Division-Multiplexing (WDM)compatible OCDMA system. This led to good encoding efficiency and correlation performance. 
J. Computer Sci., 8 (10): 1718-1729, 2012

Table 1: Summary of Hybrid WDM/OCDMA overlay schemes

\begin{tabular}{|c|c|c|c|c|c|c|}
\hline \multirow{2}{*}{$\begin{array}{l}\text { Hybrid } \\
\text { WDM/OCDMA } \\
\text { overlay schemes }\end{array}$} & \multirow{2}{*}{$\begin{array}{l}\text { Encoding } \\
\text { technique }\end{array}$} & \multirow{2}{*}{$\begin{array}{l}\text { Representative } \\
\text { code type }\end{array}$} & \multicolumn{2}{|c|}{ Modulation technique } & \multirow[b]{2}{*}{ Features } & \multirow[b]{2}{*}{ References } \\
\hline & & & WDM & OCDMA & & \\
\hline Scheme (1) & Phase & $\begin{array}{l}\text { M-sequences } \\
\text { Gold codes }\end{array}$ & OOK & OOK & $\begin{array}{l}\text { High security with } \\
\text { complex } \\
\text { Implementation }\end{array}$ & $\begin{array}{l}\text { (Chang } \text { et al., 2005; } \\
\text { Shen and Weiner, 2001; } \\
\text { Gao et al., 2010) }\end{array}$ \\
\hline Scheme (2) & Phase & Random Codes & OOK & M-ary & $\begin{array}{l}\text { High security with } \\
\text { complex } \\
\text { implementation }\end{array}$ & (Wu and Narimanov, 2006) \\
\hline Scheme (3) & WHTS & Prime-hop Codes & OOK & M-ary & $\begin{array}{l}\text { High security with } \\
\text { complex } \\
\text { implementation }\end{array}$ & $\begin{array}{l}\text { (Wu et al., 2006; } \\
\text { Kiurvtso } \text { et al., 2007) }\end{array}$ \\
\hline Scheme (4) & Phase & $\begin{array}{l}\text { Hadamard codes } \\
\text { (duobinary) }\end{array}$ & OOK & OOK & $\begin{array}{l}\text { High security with } \\
\text { complex } \\
\text { implementation }\end{array}$ & (Galli et al., 2005) \\
\hline Scheme (5) & SAC & MQC , MDW & $\mathrm{OOK}$ & OOK & $\begin{array}{l}\text { Moderate security with } \\
\text { simple implementation }\end{array}$ & $\begin{array}{l}\text { (Ashour et al., 2011a; } \\
\text { Ashour } \text { t al., 2011b) }\end{array}$ \\
\hline
\end{tabular}

Tian et al. (2007) and Teh et al. (2002), a reconfigurable optical OCDMA/dense WDM system based on quaternary phase coding gratings on some channels was demonstrated. They have employed a code-reconfigurable device for decoding based on the thermo-optic effect. In addition, continuous fixed phase-shift SSFBGs and that of the reconfigurable grating were studied (Tian et al., 2007; Teh et al., 2002). They concluded that Direct-Sequence of OCDMA based on SSFBG encoding is well-suited with WDM technology. That means, WDM channels can carry conventionally encoded data such as bipolar code sequences corresponding to "orthogonal" gold codesequences. Nevertheless, this experimental system has a relatively low spectral efficiency (Teh et al., 2002).

A new kind of coding technique was utilized for analyzing the capacity of hybrid WDM/OCDMA system (Gharaei et al., 2010). In this approach, Direct-Sequence OCDMA technique is to be added to WDM. In other words, pulse train in WDM channels is encoded for each user data bit. Hence, different users can communicate through one codeword with each other simultaneously. In a simulation setup, 32 WDM channels with minimum spacing of $25 \mathrm{GHz}$ were considered. The RZ pulse train is modulated and then encoded using the Extended Congruent Code (ECC) with four OCDMA users. The performance of the system has been investigated with considering the relevant noise sources such as MAI, beat noise and receiver noise. The results reveal that simultaneous active users in the WDM/OCDMA system is defined at conventional BER $=109$.

Some other researchers have presented hybrid WDM-OCDMA-PON as the next generation system using a network simulation. This scheme could support a large number of subscribers with acceptable BER of $10^{-12}$. In this architecture, OCDMA channels can be overlaid on WDM frequency spacing. On each WDM grid of $\lambda_{n}(n=1, N), M$ users can be employed by the unique assignment of each user with a different optical code, $\mathrm{OC}_{\mathrm{m}}(\mathrm{m}=1, \mathrm{M})$, which means that the same code sequence can be reused and transmitted on all the WDM channels. Thus, the total number of active users using the PON becomes $\mathrm{N} \times \mathrm{M}$. For example, if we have 4 different OCDMA coders with 3 WDM, then the total number of users which can be accommodated in the PON is 12 . The WDM inter-channel crosstalk and the spectral efficiency may be faced in these kinds of systems. So that, the channel spacing needs to be less than the chip-rate for decreasing the effect of cross talk. In the case of WDM channel spacing of 200 and $400 \mathrm{GHz}$, the effect of interchannel crosstalk can be negligible (Gharaei et al., 2010; Yang et al., 2010; Wang et al., 2007; Kitayama et al., 2006). The theoretical modeling has also been presented for this scheme, which is employing Differential Quadrature Phase Shift Keying (DQPSK) modulation with balanced detection. The results show that BER is $10^{-9}$ or less when 24 asynchronous users modulated at $10 \mathrm{Gbps}$ data rates. The analytical model of error probability in Passive Optical Network (PON) has been proposed when considering Optical Orthogonal Codes (OOC) for OCDMA over DWDM (Kora et al., 2009).

The performance of both hybrid WDM+OCDMA and Multi-Wavelength OCDMA (MW OCDMA) systems have been studied and compared to each other (Ji et al., 2008; Jianhua et al., 2005). OCDMA can be basically divided into one and two-dimensional systems in accordance to its spreading mode. MW OCDMA and WDM+OCDMA are among two-dimensional OCDMA systems with the best foreground. In WDM+OCDMA system, the same set of Optical Orthogonal Codes (OOCs) can be reused on each wavelength channel. While in MW OCDMA system, each address code employs a set of symmetric Prime-hop pulses with various frequencies transmitted with different velocities. That leads to chromatic dispersion. However, the WDM+OCDMA system performance is better when traffic load is heavy. 
Shifted Prime (SP) codes for SAC OCDMA-based Passive Optical Network (PON) has been proposed in (Yang, 2009) with the combination of the WDM technique. A new family code for this system has been constructed and named WS-SP. In addition, since a bidirectional WDM-PON is used to connect the OLT and the ONUs, two SAC codewords for both downstream and upstream transmission are assigned (Yang, 2009). This leads to performance improvement since the arising noise during photo-detecting process is reduced. Furthermore, it can enhance the ability against eavesdropping. The results of theoretical analysis in (Jianhua et al., 2005), demonstrates these features.

Another new family code for SAC/WDM based on OCDMA network has been proposed in (Huang et al., 2010). The codes are Extended Welch-Costas (EWC) and Quasi-Cyclic Low-Density Parity-Check (QCLDPC) code. The performance of the EWC coded OCDMA system gets better by using the LDPC codes as shown in the simulation results in (Huang et al., 2010) where this code has a superior performance as compared to the conventional SAC OCDMA systems, which was demonstrated based on the performance analysis.

However, WDM in these systems was used as a multiplexing technique or a medium for various OCDMA signals. Many types of codes and variety of architectures have been employed in accordance of this kind of hybrid scheme for mostly improving the system capacity.

\section{CONCLUSION}

Hybrid OCDMA and WDM systems have been reviewed with exploration of new architectures that support high capacity and security for optical fiber communications. In addition, several coding techniques affect the system performance and flexibility. We also provided a compact review of the fundamental background of OCDMA and security of fiber optics for new researchers in this field.

\section{ACKNOWLEDGMENT}

We would like to acknowledge Universiti Kebangsaan Malaysia (UKM) for sponsoring this project under research grant UKM-GGPM-NBT-0902010, UKM-OUP-NBT-27-119/2011 and fellowship grant -UKM Zamalah.

\section{REFERENCES}

Ab-Rahman, M.S., B. Ng, N.M. Ibrahim and S. Shaari, 2009. Low-cost encoding device for optical code division multiple access system, Am. J. Engin. Applied Sci., 2: 317-323. DOI: 10.3844/ajeassp.2009.317.323
Agrawal, G.P., 2002. Fiber-Optic Communications Systems. 3rd Ed., John Wiley and Sons Inc.

Algalbi, W.J.M., 2009. Solving the near-far problem in dynamic frequency hopping-optical code division multiple access using power control. Proceedings of the 1st International Conference on Energy, Power and Control, Nov. 30-Dec. 2, IEEE Xplore Press, Basrah, pp: 413-418.

Aljunid, S.A., M. Ismail, A.R. Ramli, B.M. Ali and M.K. Abdullah, 2004. A new family of optical code sequences for spectral-amplitude-coding optical CDMA systems. IEEE Photonics Tech. Lett., 16: 2383-2385. DOI: 10.1109/LPT.2004.833859

Ashour, I.A.M., H.M.H. Shalaby and P.S. Menon, 2011a. Performance analysis of SAC/optical CDMA and WDM under a hybrid overlay scheme. Proceedings of the 2nd International Conference on Photonics, Oct. 17-19, IEEE Xplore Press, Malaysia, $\quad$ pp: $1-4 . \quad$ DOI: 10.1109/ICP.2011.6106874

Ashour, I.A.M., S. Shaari, H.M.H. Shalaby, P.S. Menon and H.A. Bakarman, 2010. Hybrid SAC/optical CDMA-WDM overlay system for enhancing network security. Proceedings of the $1 \mathrm{st}$ International Conference on Photonics, Jul. 5-7, IEEE Xplore Press, Kedah, Malaysia, pp: 1-4. DOI: 10.1109/ICP.2010.5604393

Ashour, I.A.M., S. Shaari, P.S Menon and H.M.H. Shalaby, 2011b. Investigation of in-band transmission of both spectral amplitude coding/optical code division multiple-access and wavelength division multiplexing signals. SPIE Opt. Eng. DOI: 10.1117/1.3589296

Bakarman, H.A., S. Shaari and M. Ismail, 2008. Security performance of spectral amplitude coding OCDMA systems. Proceedings of the IEEE International Conference Electronic Design, Dec. 1-3, IEEE Xplore Press, Bangi, DOI: 10.1109/ICED.2008.4786740

Bakarman, H.A., S. Shaari and M. Ismail, 2009. Security performance of spectral amplitude code OCDMA: Spectrally encoded pulse bandwidth effects. J. Opt. Commun., 30: 242-247. DOI: 10.1515/JOC.2009.30.4.242

Cederlof, J. and J.A. Larsson, 2008. Security aspects of the authentication used in quantum cryptography. IEEE Trans. Inform. Theory, 54: 1735-1741. DOI: 10.1109/TIT.2008.917697

Chang, P.H., F.S. Ting and J.R. Chen, 2005. Asynchronous hybrid optical code division/wavelength division multiple access system. Proceedings of the 3rd International Conference on Information Technology: Research and Education, Jun. 27-30, IEEE Xplore Press, pp: 131-135. DOI: 10.1109/ITRE.2005.1503083 
Chen, C., 2005. Top 10 unsolved information visualization problems. IEEE Comput. Graphics Applic., 25: 12-16. DOI: 10.1109/MCG.2005.91

Etemad, S., P. Toliver, R. Menendez, J. Young and T. Banwell et al., 2005. Spectrally efficient optical CDMA using coherent phase-frequency coding. IEEE Photonic Technol. Lett., 17: 929-931. DOI: 10.1109/LPT.2005.843692

Fouli, K. and M. Maier, 2007. OCDMA and Optical Coding: Principles, Applications and Challenges. IEEE Commun. Mag., 45: 27-34. DOI: 10.1109/MCOM.2007.4290311

Galli, S., R. Menendez, P. Toliver, T. Banwell and J. Jackel et al., 2005. Experimental results on the simultaneous transmission of two 2.5 Gbps opticalCDMA channels and a 10 Gbps OOK channel within the same WDM window. Proceedings of the Technical Digest. OFC/NFOEC Optical Fiber Communication Conference, Mar. 6-11, IEEE Xplore Press, USA. DOI: 10.1109/OFC.2005.192704

Gao, Z., X. Wang, N. Kataoka and N. Wada, 2010. Stealth transmission of time domain spectral phase encoded OCDMA signal over WDM system. Proceedings of the Collocated National Fiber Optic Engineers Conference on Optical Fiber Communication, Mar. 21-25, IEEE Xplore Press, San Diego CA., pp: 1-3.

Gharaei, M., C. Lepers and P. Gallion, 2010. Impact of crosstalk in capacity performance of WDM/OCDMA system. Proceedings of the Collocated National Fiber Optic Engineers Conference on Optical Fiber Communication, Mar. 21-25, IEEE Xplore Press, San Diego, CA., pp: 1-3.

Hamam, H., 2006. Optical Fiber Components: Design and Applications. 1st Edn., Transworld Research Network, India, ISBN: 8130800977, pp: 243.

Hassan, A.A., J.E. Hershey and N.A. Riza, 1995. Spatial optical CDMA. IEEE J. Selected Areas Commun., 13: 609-613. DOI: 10.1109/49.372419

Hata, M., N. Shiraishi, S. Tomisato, 2006. Cellular CDMA system capacity in a service area with tapered traffic distribution. Proceedings of the IEEE Wireless Communications and Networking Conference, Apr. 3-6, IEEE Xplore Press, Las Vegas, NV, pp: 25-29. DOI: 10.1109/WCNC.2006.1683435

Huang, C.M., J.F. Huang and C.C. Yang, 2010. Performance analysis of WS-EWC coded optical CDMA networks with/without LDPC codes. Optical Fiber Technol., 16: 265-270. DOI: 10.1016/j.yofte.2010.05.004
Huang, J.F., Y.T. Chang, C.C. Sue and C.C. Hsu, 2006. Hybrid WDM and optical CDMA implemented with $\mathrm{m}$-sequence coded waveguide gratings over fiber-to-the-home network. Proceedings of the Communications, Circuits and Systems, Jun. 2528, IEEE Xplore Press, Guilin, pp: 1860-1864. DOI:10.1109/ICCCAS.2006.285035

Hui, J.Y., 1985. Pattern code modulation and optical decoding-a novel code-division multiplexing technique for multifiber networks. IEEE J. Sel. Areas Commun., 3: 916-927. DOI: 10.1109/JSAC.1985.1146265

Ji, J., Z. Chen and M. Xu, 2008. Performance Analysis of WDM-based Coherent Time-Spreading Optical CDMA System. Proc. of SPIE 7137: 713-731.

Jiang, Z., D. Seo, S. Yang, D.E. Leaird and R.V. Roussev et al., 2005. Four-user 10-Gb/s spectrally phase-coded O-CDMA system operating at $\sim 30$ fJ/bit. IEEE Photon. Technol. Lett. 17: 705-707.

Jianhua, J., Y. Shuwen, X. Ming and Z. Zhipeng. 2005. Performance comparison of WDM+OCDMA and multi-wavelength OCDMA systems. Proceedings of the 3rd Optical Transmission, Switching and Subsystems. (OTSS' 25), SPIE, pp: 912-915.

Kitayama, K., X. Wang and N. Wada, 2006. OCDMA Over WDM PON-Solution Path to GigabitSymmetric FTTH. J. Lightwave Technol., 24: 1654-1662. DOI: 10.1109/JLT.2006.871030

Kiurvtso, K., P.R. Prucnal, E. Nanimanov, 2007. Stealth transmission over a WDM network with detection based on an all-optical thresholder. IEEE Proceedings of the 20th Annual Meeting of Lasers and Electro-Optics Society, Oct. 21-25, IEEE Xplore Press, Princeton, pp: 480-481. DOI: 10.1109/LEOS.2007.4382488

Kora, A.D., F. Diop, J.P. Cances and S. Ouya, 2009. Optical orthogonal codes over wavelength division multiplexing in passive optical network communication system and its Performance analysis. Proceedings of the Adaptive Science and Technology, Jan. 14-16, IEEE Xplore Press, Senegal, pp: 40-44. DOI: 10.1109/ICASTECH.2009.5409750

Kwong, W.C., G.C. Yang and C.Y. Chang, 2005. Wavelength-hopping time-spreading optical CDMA with bipolar codes. J. Lightwave Technol., 23: 260-267. DOI: 10.1109/JLT.2004.835748(410) 23

Lee, Y. and S. Kim, 2000. Sequence Acquisition of DSCDMA systems employing gold sequences. IEEE Trans. Vehicular Tech., 49: 2397-2404. DOI: 10.1109/25.901908

Leeson, M.S., K. Cui and E.L. Hines, 2009. Enhancing optical CDMA. Proceedings of the 11th International Conference on Transparent Optical Networks, Jun. 28-Jul. 02, IEEE Xplore Press, Azores, pp: 362-365. DOI: 10.1109/ICTON.2009.5185306 
Lam, A., 2000. Tacit knowledge, organizational learning and societal institutions: An integrated framework. Organiz. Stud., 21: 487-513. DOI: 10.1177/0170840600213001

Mohammed, A., N.M. Saad, E.I. Babekir, N. Elfadel and S.A. Aljunid et al., 2007. Modeling and simulation of the double weight code family detection schemes. Proceedings of the International Conference on Intelligent and Advanced Systems, Nov. 25-28, IEEE Xplore Press, Tronoh, pp: 348351. 10.1109/ICIAS.2007.4658405

Pickholtz, R., D. Schilling and L. Milstein, 1982. Theory of spread-spectrum communications--a tutorial. IEEE Trans. Commun., 30: 855-884. 10.1109/TCOM.1982.1095533

Prucnal, P., M. Santoro and T. Fan, 1986. Spread spectrum fiber-optic local area network using optical processing. J. Lightw. Technol., 4: 547-554. DOI: 10.1109/JLT.1986.1074754

Pu, T., Y. Zhu, P. Chen, X. Chen and L. Lu et al., 2007. Novel encoder/decoder using subsampled bragg grating for a WDM-compatible OCDMA system. IEEE Photonics Technol. Lett., 19: 1807-1809. DOI: 10.1109/LPT.2007.906843

Sahbudin, R.K.Z., M.K. Abdullah, M. Mokhtar, S. Hitam and S.B.A. Anas, 2011. Design and cost performance of decoding technique for hybrid subcarrier spectral amplitude coding-optical code division multiple access system. J. Comput. Sci., 7: 1525-1531. DOI: 10.3844/jcssp.2011.1525.1531

Salehi, J.A. and C.A. Brackett, 1989. Code division multiple-access techniques in optical fiber networks. II. Systems performance analysis. IEEE Trans. Commun., 37: 834-842. DOI: $10.1109 / 26.31182$

Salehi, J.A., 1989. Code division multiple-access techniques in optical fiber networks. I. Fundamental principles. IEEE Trans. Commun., 37: 824-833. DOI: 10.1109/26.31181

Sangin, K., K. Yu and N. Park, 2000. A new family of space/wavelength/time spread three-dimensional optical code for OCDMA networks. J. Lightwave Technol., 18: 502-511.

Shake, T.H. 2005. Security performance of optical CDMA Against eavesdropping. J. Lightwave Technol., 23: 655-670. DOI: 10.1109/JLT.2004.838844

Shen, S. and A.M. Weiner, 2001. Suppression of WDM interference for error-free detection of ultrashortpulse CDMA signals in spectrally overlaid hybrid WDM-CDMA operation. IEEE Photonics Techno. Lett., 13: 82-84. DOI: 10.1109/68.903228

Smith, E.D., R.J. Blaikie and D.P. Taylor, 1998. Performance enhancement of spectral-amplitudecoding optical CDMA using pulse-position modulation. IEEE Trans. Commu., 46: 1176-1185. DOI: $10.1109 / 26.718559$
Sotobayashi, H., W. Chujo and K. Kitayama, 2004. Highly spectral-efficient optical code-division multiplexing transmission system. IEEE J. Selected Top. Quantum Elec., 10: 250-258. DOI: 10.1109/JSTQE.2004.826568

Stok, A. and E.H. Sargent, 2002. The role of optical CDMA in access networks. IEEE Commun. Mag., 40: 83-87. DOI: 10.1109/MCOM.2002.1031833

Tamura, S., S. Nakano and K. Okazaki, 1985. Optical code-multiplex transmission by Gold sequences. J. Lightw. Technol., 3: 121-127. DOI: 10.1109/JLT.1985.1074148

Teh, P.C., M. Ibsen, J.H. Lee, P. Petropoulos and D.J. Richardson, 2002. Demonstration of a four-channel WDM/OCDMA system using 255-chip 320Gchip/s quarternary phase coding gratings. IEEE Photonics Techno. Lett., 14: 227-229. DOI: 10.1109/68.980530

Tian, C., Z. Zhang, M. Ibsen, P. Petropoulos and D.J. Richardson, 2007. A 16-channel reconfigurable OCDMA/DWDM System Using Continuous Phase-Shift SSFBGs. IEEE J. Selected Topics in Quantum Elec., 13: 1480-1486. DOI: 10.1109/JSTQE.2007.897668

Viterbi, A., 1979. Spread spectrum communications-Myths and realities. IEEE Commun. Mag., 17: 11-18. DOI: 10.1109/MCOM.1979.1089969

Wang, X., 2008. Recent progresses in OCDMA. Proceedings of the 10th Anniversary International Conference on Transparent Optical Networks, June 22-26, IEEE Xplore Press, Edinburgh, pp: 39-42. DOI: 10.1109/ICTON.2008.4598365

Wang, X., K. Matsushima, A. Nishiki, N. Wada and K. Kitayama, 2004. High reflectivity superstructured FBG for coherent optical code generation and recognition. Optics Express, 12: 5457-5468. DOI: 10.1364/OPEX.12.005457

Wang, X., K. Matsushima, A. Nishiki, N. Wada, F. Kubota and K. Kitayama, 2005. High performance optical code generation and recognition using 511 chip 640Gchip/s phase-shifted superstructureed FBG. Optics Lett., 30: 355-357.

Wang, X., N. Wada, G. Cincotti, T. Miyazaki and K. Kitayama, 2006. Demonstration of over $128-\mathrm{gb} / \mathrm{s}-$ capacity (12-User/spl times/10.71-gb/s/user) asynchronous OCDMA using FEC and AWGbased multiport optical encoder/decoders. IEEE Photonics Technol. Lett., 18: 1603-1605. DOI: 10.1109/LPT.2006.879594

Wang, X., N. Wada, T. Miyazaki, G. Cincotti and K. Kitayama, 2007. Hybrid WDM/OCDMA for next generation access network. Proceedings of the SPIE, Optical Transmission, Switching and Subsystems, Nov. 1-1 SPIE Digital Library, China. DOI: $10.1117 / 12.745673$ 
Wei, Z., H.M.H. Shalaby and H. Ghafouri-Shiraz, 2001. Modified quadratic congruence codes for fiber Bragg-grating-based spectral-amplitude-coding optical CDMA systems. J. Lightwave Technol., 19: 1274-1281. DOI: $10.1109 / 50.948274$

Wu, B.B. and E.E. Narimanov, 2006. A method for secure communications over a public fiber-optical network. Optics Express, 14: 3738-3751. DOI: 10.1364/OE.14.003738

Wu, B.B., P.R. Prucnal and E.E. Narimanov, 2006. Secure transmission over an existing public WDM lightwave network. IEEE Photonics Technol. Lett., 18: 1870-1872. DOI: 10.1109/LPT.2006.881234

Yang, C.C., 2005. Hybrid wavelength-divisionmultiplexing/spectral-amplitude-coding optical CDMA system. IEEE Photonics Technol. Lett., 17: 1343-1345. DOI: 10.1109/LPT.2005.847447

Yang, C.C., 2009. High speed and secure optical CDMA-based passive optical networks. Computer Networks, 53: 2182-2191. DOI: 10.1016/j.comnet.2009.04.004
Yang, L., H. Zhang, X. Zheng, B. Zhou and Y. Guo et al., 2010. Next generation hybrid OCDMA-WDMPON with Soft capacity. Proceedings of the 15th OptoeElectronics and Communications Conference, July 5-9, IEEE Xplore Press, China, pp: 732-733.

Yegnanarayanan, S., A.S. Bhushan and B. Jalali, 2000. Fast wavelength-hopping time-spreading encoding/decoding for optical CDMA. IEEE Photonics Technol. Lett., 12: 573-575.

Yim, R.M.H., L.R. Chen and J. Bajcsy, 2002. Design and performance of 2-D codes for wavelength-time optical CDMA. IEEE Photonics Technol. Lett., 14: 714-716. DOI: $10.1109 / 68.998735$

Yin, H., D.J. Richardson, 2007. Optical Code Division Multiple Access Communication Networks: Theory and Applications. 1st Edn., Tsinghua University Press, Beijing, ISBN: 9783540684459, pp: 382. 